(C) 2017 IEEE. Personal use of this material is permitted. Permission from IEEE must be obtained for all other uses, in any current or future media, including reprinting/republishing this material for advertising or promotional purposes, creating new collective works, for resale or redistribution to servers or lists, or reuse of any copyrighted component of this work in other works. 


\title{
Robust Extreme Value Estimation for Full Time-Domain EMI measurements
}

\author{
Marco A. Azpúrua, José A. Oliva, Marc Pous, Ferran Silva \\ Grup de Compatibilitat Electromagnètica (GCEM), Departament d'Enginyeria Electrònica (DEE) \\ Universitat Politècnica de Catalunya (UPC) \\ Barcelona, Spain \\ email: marco.azpurua@upc.edu
}

\begin{abstract}
A robust approach for estimating the expected maximum levels of radiofrequency, time-varying, electromagnetic emissions is proposed. The expected maximum peak value is intended to provide a statistical approximation for the worst case emissions scenario that accounts for the variability of the measured interference. The estimates are obtained through Monte Carlo resampling from a non-parametric distribution fitted by means of kernel density estimation applied to the time-frequency representation of the assessed interference. As a key advantage, calculating the expected maximum peak value does not require increasing the dwell time or holding the maximum value over successive sweeps. Results indicate the methodology is better suited than previous approaches for calculating the expected maximum peak value because it does not depend on normality assumptions difficult to guarantee in practice. The proposed technique is an example of how full time-domain EMI measurements can be exploited for obtaining further insights.
\end{abstract}

Keywords- Statistical signal processing, Extreme value estimation, Electromagnetic interference, Electromagnetic measurements, Time-domain analysis

\section{INTRODUCTION}

In emission testing, the electromagnetic disturbance spectrum measured with the receiver's peak detector mode is intended to provide a fast realization of the worst-case scenario with regard the interferences produced by the equipment under test (EUT). In swept receivers, increasing the dwell time enables a better approximation to the actual maximum emissions. Likewise, whenever the EUT generates a highly variable electromagnetic interference (EMI), it is the common practice to increase the number of sweeps and to retain the maximum observation for each frequency step, that is, the "max-hold" feature included in most test receivers.

The aforementioned procedure certainly increases the probability of measuring the worst-case emissions, but still can be heavily time consuming if such worst-case scenario is due to an interference of low repetition frequency. In such cases, the triggering capabilities of Full Time Domain EMI measurement systems (Full TDEMI) can be especially helpful for detecting the highest EMI event (in the time domain) and capturing its whole spectrum in a single acquisition [1], [2].

Despite the advantages provided by the trigger in Full TDEMI measurement systems, it is likely that a significantly long dwell time is still required to measure properly the highest
EMI levels at each frequency step. However, increasing indefinitely the dwell time is not feasible. For instance, the memory of the oscilloscope limits the maximum dwell time settable in Full TDEMI measurement systems [3]. Additionally, the blind time between successive deep memory acquisitions and the potentially enormous amount of raw data generated poses constraints to the maximum dwell time, and therefore, to the capability of the measurement system to record EUT's highest emissions.

Nonetheless, time-domain EMI measurements enable the extraction of statistical information about the variability of the disturbance. Such information is useful for calculating the expected maximum emissions levels of the EUT under assessment. In this regard, in a previous paper, the authors investigated the statistical properties of the peak detector in time-domain EMI measurements. Using the extreme value theory, the maximum emission levels were modeled as random variables with Gumbel distribution, and the expressions to evaluate its parameters, expected value, variance and CramerRao bounds were provided [4].

In this regard, the expected value and the variance of the maximum electromagnetic emissions were calculated provided the measured interference is normally distributed at each frequency step. On the other hand, the assumption of normality is not necessarily satisfied, as happens commonly with the impulsive noise [5]. Thus, when the EMI magnitude at each frequency bin is not approximately normally distributed, a robust approach to the extreme value estimation is required in order to provide fair estimations of the expected maximum emissions.

This paper presents a numerical approach for the robust estimation of the expected maximum electromagnetic emissions measured with Full TDEMI measurement systems. The content is organized as follows: Section II introduces Full TDEMI measurement systems; Section III presents the methodology for performing a robust extreme value estimation of the EMI; Section IV focuses on a measurement example employed to compare to the results obtained using the analytical approach and, finally, the conclusions.

\section{FULL TDEMI MEASUREMENT SYSTEMS}

Full TDEMI measurement systems are oscilloscope based implementations of a CISPR 16-1-1 compliant measuring 
receiver [6]. According to the CISPR standards, they are a type of FFT-based instruments. Unlike real-time EMI receivers, Full TDEMI measurement systems do not have an intermediate frequency stage and the measured frequency range is not divided into sub-bands. That is the fundamental reason why the whole (full) spectrum is measured in each acquisition, which is a key capability for measuring transient EMI. In general, they require digitizing the EMI using much higher sampling rates than other types of FFT-based receivers causing full-spectrum measurements to be memory intensive when standard dwell times are used.

After deep memory acquisition, the software of the Full TDEMI measurement system performs signal processing tasks including windowing, resolution enhancing, resampling, spectral estimation (using the Short-Time Fourier Transform and the Welch's method) and the detector emulation [2]. Those mathematical transformations are responsible for delivering the measurement results in accordance with CISPR 16-1-1 requirements [7].

In general terms, a Full TDEMI measurement system is described by the block diagram shown in Fig. 1. For the measurement of radiated EMI, a broadband antenna shall be used, while for the measurement of conducted EMI corresponds either a line impedance stabilization network (LISN), a capacitive voltage probe or a current clamp. The measured signal could be amplified and/or filtered if better sensitivity is required [2].

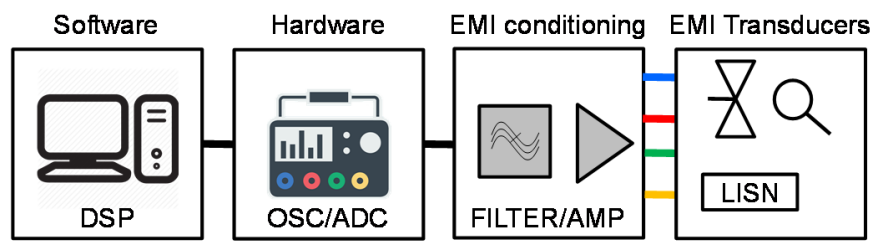

Fig. 1. Block diagram of a Full Time-Domain EMI measurement system [2].

\section{ROBUST EXTREME VALUE ESTIMATION FOR EMI MEASUREMENTS}

In this section, the fundamental signal processing techniques and the statistical considerations used to estimate robustly the maximum emissions level will be covered in the following subsections.

\section{A. Fundamental signal processing}

First, let us consider the continuous-time signal $x(t)$ that represents the EMI under assessment. The measurement process begins when $x(t)$ is digitized and sampled during a time period of length $T$, at a rate $f_{s}=N / T_{0}=1 / \Delta t$, obtaining the time-discrete signals $x[n]$, where $n=1,2, \ldots N$. The digitized EMI signal, $x[n]=x(n \Delta t)$, is assumed to be periodic with the total number of waveform points, which means,

$$
x[n+N]=x[n] .
$$

Due to the random behavior of EMI, the former assumption of periodicity is not strictly satisfied. However, this is acceptable provided a sufficiently large dwell time with respect to the EMI repetition frequency, that is, $T_{0} \gg 1 / f_{r}$. With regard the sampling rate, it must be enough to satisfy the Nyquist criterion and to avoid aliasing [2].

Then, the digitized EMI is transformed to the frequency domain using the Discrete Fourier Transform (DFT) as follows,

$$
X[k]=\sum_{n=0}^{N-1} x[n] e^{-i \frac{2 \pi n k}{N}} .
$$

However, in order to reduce the scalloping loss and the spectral leakage caused by the finite length the time record, $x[n]$ must be multiplied by a window function $w[n]$. It shall be noted that the windowing function is such that its frequency response suits the IF filter requirements of an EMI receiver [8]. If windowing is used, it is necessary to compensate the corresponding energy loss caused by the window using the coherent gain scaling factor [9].

Consequently, to obtain a spectral estimation with the required resolution bandwidth, RBW (that is, $200 \mathrm{~Hz}, 9 \mathrm{kHz}$, $120 \mathrm{kHz}$ and $1 \mathrm{MHz}$ for CISPR bands A, B, C/D and, E, respectively), the minimum record length, $N_{\min }$, shall be

$$
N_{\text {min }}=f_{s} \frac{w_{f}}{\mathrm{RBW}},
$$

where $w_{f}$ is the window factor of the windowing function used, i.e., Gaussian, Hann or Kaiser-Bessel [2].

If $N>N_{\min }$, it is possible to define $J$ overlapping windows of $N_{\min }$ length in order to determine the frequency and phase content of the $j$-th local sections of $x[n]$ as it changes over time. This process, called the Short-Time Fourier Transform (STFT), can be expressed as

$$
X[k, \tau]=\sum_{n=0}^{N_{\min }-1} x[n-\tau] w[n] e^{-i \frac{2 \pi n k}{N_{\min }}},
$$

where $\tau$ describes the dependency on time of each spectral component of the spectrogram [10]. Consequently, the spectrogram time steps of the STFT are given by

$$
t_{\text {steps }}=\tau \Delta t=j\left(1-o_{f}\right) N_{\min } \Delta t,
$$

for $j=1,2, \ldots J$. Likewise, the spectrogram frequency bins are $f_{\text {bins }}=k \Delta f$, where $\Delta f$ is the frequency resolution. An adequate overlap factor, $o_{f}$, must be used to reduce amplitude error caused by the scalloping loss up to an acceptable level [11], [12].

Subsequently, the STFT can be rearranged in a matrix form with the values of each spectral component in the rows and as the time steps in the columns, that is,

$$
\mathbf{X}[k, j]=\left[\begin{array}{cccc}
X[1,1] & X[1,2] & \cdots & X[1, J] \\
X[2,1] & X[2,2] & \cdots & X[2, J] \\
\vdots & \vdots & \ddots & \vdots \\
X\left[k_{\max }, 1\right] & X\left[k_{\max }, 2\right] & \cdots & X\left[k_{\max }, J\right]
\end{array}\right] .
$$

Considering (6) provides the information of the timefrequency distribution of $x(t), \mathbf{X}[k, j]$ can be used for calculating the different detector modes required for standard 
EMI measurements [4], [12], [13]. In the particular case of the peak detector, $X_{\text {peak }}[k]$, it corresponds to,

$$
X_{\text {peak }}[k]=\max \{|\mathbf{X}[k, j]| \mid j \in\{1,2, \ldots J\}\},
$$

\section{B. Distribution analysis}

Expressions for the expected maximum EMI levels will be presented. The forthcoming analysis assumes the EMI under assessment behaves as a (cyclostationary) random signal and, that the measured time-frame is an independent and representative sample of the interference under assessment.

In this regard, the windowed time frames obtained by the STFT (6) are a group of subsamples from which the EMI spectral content was estimated $J$ times, one spectral estimation from each subsample. The variations of the spectral content at each frequency bin are then analyzed in terms of random variables. This means $X_{\text {peak }}[k]$ is an estimator of the extreme value taken by each component of the amplitude spectrum.

If $G_{\max }(X[k])$ is the cumulative distribution of $X_{\text {peak }}[k]$,

$$
G_{\max }(X[k])=P\left(X_{\text {peak }}[k] \leq X[k]\right),
$$

and, considering independence between the outcomes of the spectral estimation of each individual subsample, and also, that each individual subsample is identically distributed as $F(X[k])$ since they belong to the same population, then,

$$
G_{\max }(X[k])=\prod_{j=1}^{J} P\left(X_{j}[k] \leq X[k]\right)=[F(X[k])]^{J} .
$$

Finally, the probability density function (PDF) of $X_{\text {peak }}[k]$ is given by the derivative of $G_{\max }(X[k])$, that is,

$$
X_{\text {peak }}[k] \sim J[F(X[k])]^{J-1} f(X[k]),
$$

where $f(X[k])$ is the PDF of $X[k]$. This means the PDF of the maximum of each spectral component depends directly on the sample size and on the distribution function of the spectral component.

Unfortunately, the distribution function of each spectral component is generally unknown. However, the generalized extreme value (GEV) distribution allows modeling the largest (and the smallest) value among a large set of independent, identically distributed random values [14], therefore, $X_{\text {peak }}[k] \sim f_{G E V}(X[k] ; \alpha[k], \beta[k], \xi[k])$. In that sense, the GEV distribution is given by,

$$
f_{G E V}(X ; \alpha, \beta, \xi)=\frac{1}{\beta} \exp \left(-\left(1+\xi \frac{(X-\alpha)}{\beta}\right)^{-\frac{1}{\xi}}\right)\left(1+\xi \frac{(X-\alpha)}{\beta}\right)^{-1-\frac{1}{\xi}}
$$

where $\alpha, \beta$ and, $\xi$ are called the location, scale and, shape parameters respectively. It is important to notice that the dependency with the discrete frequency, $k$, has been intentionally omitted from (11) for a better readability of the equation.

\section{Parameter estimation}

In a previous work [4], the above mentioned GEV distribution was simplified to the $\xi=0$ case, called Gumbel distribution in order to obtain an analytic expression of the location and scale parameters under the assumption that $X[k]$ is normally distributed.

Nonetheless, for the generality of cases, such normality could not be granted and a robust calculation methodology must be applied. However, from each EMI measurement there is a single peak value per frequency step and, consequently, it is not possible to perform parameter estimation based on a sample of maximum values.

In this regard, a numerical approach based on nonparametric distribution fitting and resampling techniques is proposed. In order to proceed, the first step is to create a nonparametrical model for the distribution of $X[k]$ using kernel density estimation (KDE), using (12) [15]. In particular, a Gaussian kernel is used (13) [15], that is,

$$
X[k] \sim f(X[k]) \approx \frac{1}{J h} \sum_{j=1}^{J} K\left(\frac{X[k]-X[k, j]}{h}\right),
$$

where $K(\bullet)$ is the kernel function and $h>0$ is the smoothing parameter called bandwidth, which are given by,

$$
\begin{gathered}
K(u)=\frac{1}{2 \pi} e^{-\frac{1}{2} u^{2}}, \text { and }, \\
h=\left(0.9 J^{-1 / 5}\right) \min \{\hat{\sigma}, \mathrm{IQR} / 1.34\},
\end{gathered}
$$

where $\hat{\sigma}$ is the standard deviation of the sample and the IQR is the interquartile range. The criteria for choosing $h$ was selected because it provides good performance for both unimodal and bimodal densities in terms of the mean integrated square error[15].

Then, a large number of equally sized samples distributed according to (12) is generated using a Monte Carlo approach. This means, a number $M \geq 1000$ of samples having each $J$ pseudorandom elements approximately distributed as $X[k]$ was computationally generated. Next, (7) is applied to each sample in order to create a set, $S_{\text {peak }}[k]$, of maximum values for each frequency step.

$$
S_{\text {peak }}[k]=\left\{X_{\text {peak }, 1}[k], X_{\text {peak }, 2}[k], \ldots X_{\text {peak }, M}[k]\right\},
$$

Finally, $\alpha, \beta$ and, $\xi$ are estimated using the Maximum Likelihood Estimation (MLE) method for each frequency step.

\section{Expected value for the maximum EMI levels}

It can be demonstrated that, for $X_{\text {peak }}[k] \sim f_{\text {GEV }}(X[k] ; \alpha[k], \beta[k], \xi[k])$, the expected value for the maximum EMI levels is,

$$
E\left(X_{\text {peak }}\right)=\left\{\begin{array}{cl}
\alpha+\beta(\Gamma(1-\xi)-1) / \xi & \text { for } \xi \neq 0, \xi<1 \\
\alpha+\beta \gamma & \text { for } \xi=0 \\
\infty & \text { for } \xi \geq 1
\end{array}\right.
$$


where $\gamma$ is the Euler-Mascheroni constant $(\gamma \approx 0.5772), \Gamma(\bullet)$ is the gamma function and the dependency with the discrete frequency, $k$, has been omitted for clarity.

\section{APPLICATION EXAMPLE}

This section comprises the measurement methodology and an application example relevant for showing the differences between the estimations performed using the robust and the classical approaches.

\section{A. Test setup}

The specific Full TDEMI measurement system that serves as measuring receiver is based on a real-time sampling digital storage oscilloscope Tektronix DPO5104B. A detailed explanation of the features of this Full TDEMI measurement system is found in [3].

In the same manner, an explicit description of the signal processing techniques applied to the measured signal is given in [2]. Measurements were performed using a standard setup for radiated emissions assessment inside a full anechoic chamber.

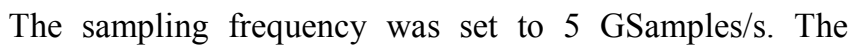
resolution bandwidth was configured at $120 \mathrm{kHz}$ and the dwell time was set to $10 \mathrm{~ms}$. The measurement distance was $3 \mathrm{~m}$ and a Schaffner CBL6143 bilog antenna in horizontal polarization was used. The EMI was generated by a pair of personal computers while transferring a large video file through an Ethernet link.

\section{B. Measurement results}

Fig. 2 shows the EMI measurement results obtained by using the standard peak and CISPR average detectors. The weighting caused by the average detector responds to the fluctuation of the EMI levels within the measured time-frame.

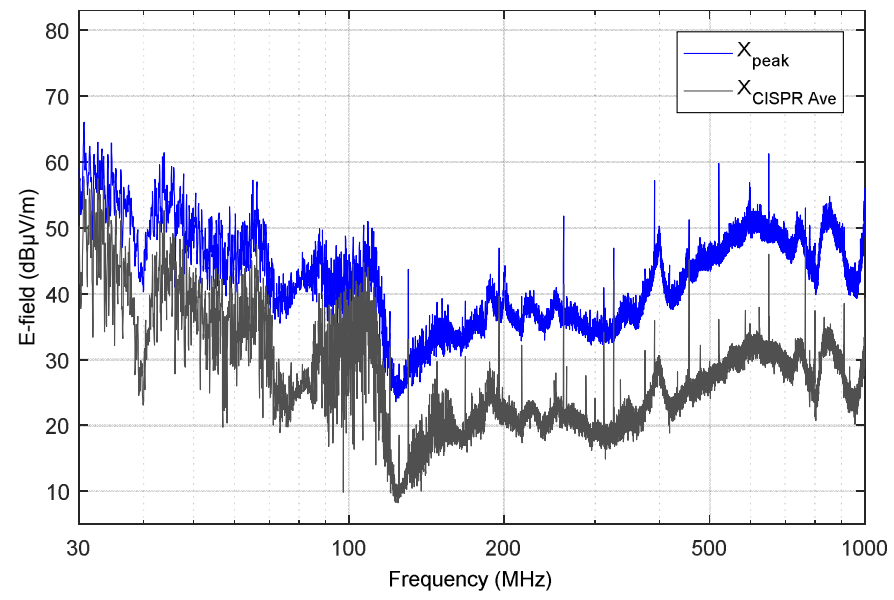

Fig. 2. EMI measurement results with the standard peak and CISPR average detectors.

The time-frequency response of the measured EMI is shown in Fig. 3 spectrogram. On the one hand, it is observed that most components of the lower frequency EMI spectrum are cyclic and stationary. However, above $500 \mathrm{MHz}$ the highest emissions occur during a $3 \mathrm{~ms}$ burst-like event.

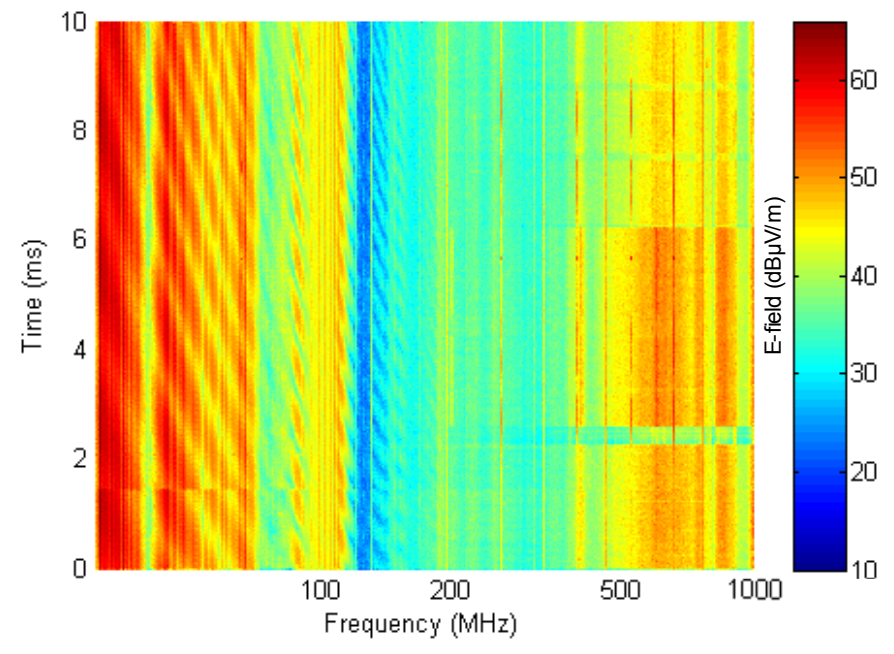

Fig. 3. Spectrogram of the measured EMI.

Likewise, the persistence plot of the measured EMI is useful to realize how the interference varies in amplitude. In that sense, Fig. 4 shows in red tones the most probable amplitude levels and fades to blue tones for the less observed values.

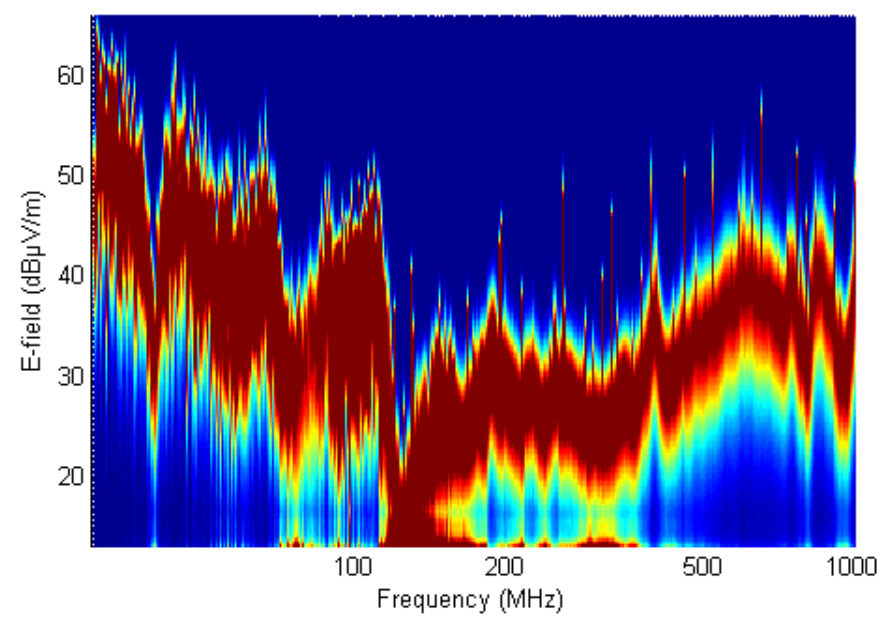

Fig. 4. Persistence plot of the measured EMI.

\section{Extreme value analysis}

Given the variability observed in the measurement results (Fig. 4), the actual maximum emissions levels could be even higher than the measured peak values. To investigate this, first, a distribution analysis was carried out. In this regard, the amplitude probability distribution of the EMI at three different frequencies is presented, as an example, and their maximum peak values are then estimated using the proposed methodology. Those frequencies are $\mathrm{f}_{1}=30.63 \mathrm{MHz}$, $\mathrm{f}_{2}=260.6 \mathrm{MHz}$ and, $\mathrm{f}_{3}=947.3 \mathrm{MHz}$.

Fig. 5 presents the probability density function fitted for the EMI amplitude at the aforementioned frequencies. It also shows the measured peak value and the expected maximum peak value with the triangle and diamond markers, respectively. It is remarkable how the distribution of the interference changes with frequency. However, at the selected frequencies, the amplitudes have a bell-shaped distribution. 


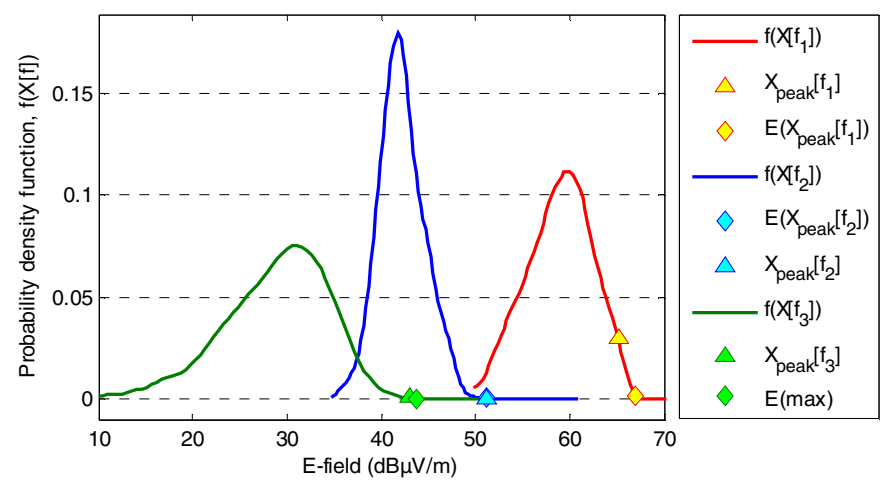

Fig. 5. Estimated probability density functions for the amplitude of the measured EMI at the selected frequencies: $f_{1}=30.63 \mathrm{MHz}, \mathrm{f}_{2}=260.6 \mathrm{MHz}$ and $\mathrm{f}_{3}=947.3 \mathrm{MHz}$.

By using the normal probability plot shown in Fig. 6, some remarkable characteristics of the distributions come to light. For instance, the amplitude of some frequency components can be skewed, as happens with $X\left[\mathrm{f}_{3}\right]$, or the shape of the distribution tails (fat/short) may depart from the normal distribution which would lead to inaccurate estimations based on the approach that assumes normality [16].

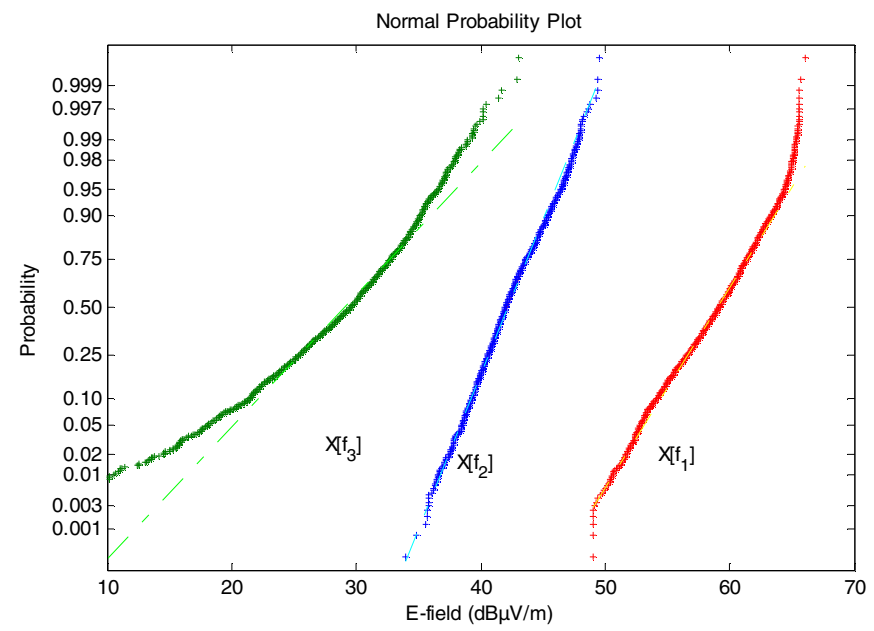

Fig. 6. Normal probability plot for the amplitude of the measured EMI at the selected frequencies.

Consequently, significant differences arises when estimating the maximum peak EMI values depending on the selected approach, as shown in Table I.

TABLE I. COMPARISON OF THE ROBUST AND THE CLASICAL EXPECTED PEAK VALUES AT THE SELECTED FREQUENCIES

\begin{tabular}{|c|c|c|c|c|}
\hline \multicolumn{2}{|c|}{$\begin{array}{c}\text { Frequency } \\
{[\mathrm{MHz}]}\end{array}$} & $\begin{array}{c}X_{\text {peak }} \\
{[\mathrm{dB} \mu \mathrm{V} / \mathrm{m}]}\end{array}$ & $\begin{array}{c}\mathrm{E}\left(X_{\text {peak }}\right) \\
{[\mathrm{dB} \mu \mathrm{V} / \mathrm{m}]}\end{array}$ & $\begin{array}{c}\text { Robust } \mathrm{E}\left(X_{\text {peak }}\right) \\
{[\mathrm{dB} \mu \mathrm{V} / \mathrm{m}]}\end{array}$ \\
\hline $\mathrm{f}_{1}$ & 30.63 & 65.16 & 70.72 & 67.01 \\
\hline $\mathrm{f}_{2}$ & 260.6 & 51.61 & 51.66 & 51.61 \\
\hline $\mathrm{f}_{3}$ & 974.3 & 42.46 & 49.35 & 43.26 \\
\hline
\end{tabular}

Likewise, the differences in the expected peak values obtained with the classical and with the robust approach are evident when represented in the whole frequency range, as shown in Fig. 7.

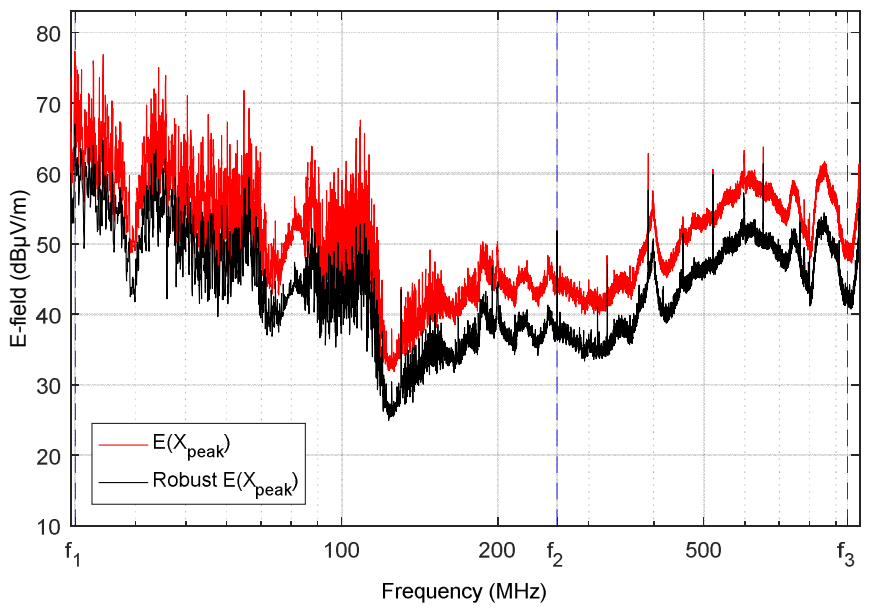

Fig. 7. Comparison of the robust and the clasical expected peak EMI values.

In general, the robust approach delivered more conservative and reasonable estimates. By using the robust estimation, approximately $80 \%$ of the frequency components are expected to have a maximum peak value that is less than $1 \mathrm{~dB}$ higher than the actual measured value, as shown in Fig 8.

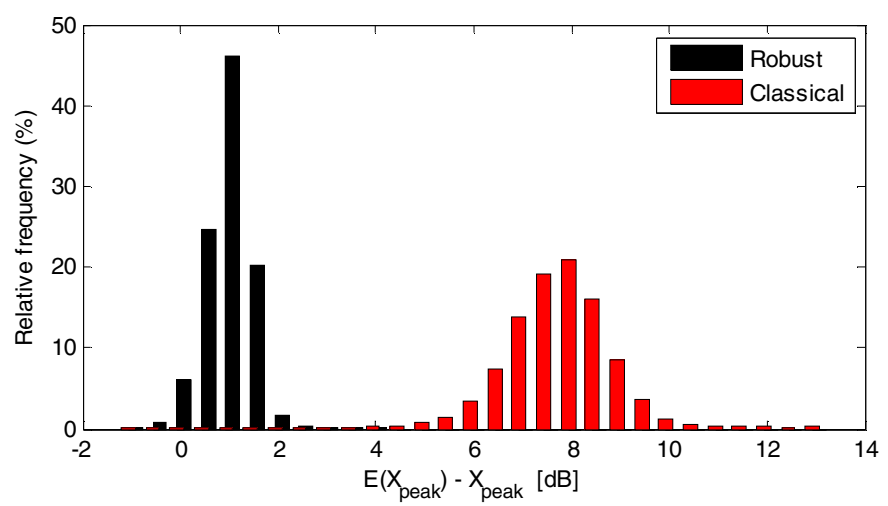

Fig. 8. Histograns of relative frequency for the difference between the measured and the expected peak values.

Likewise, according to the robust estimation, the maximum difference encountered between the expected peak and the measured peak was $3.78 \mathrm{~dB}$ at $\mathrm{f}_{4}=43.17 \mathrm{MHz}$. Analyzing the distribution of the EMI at that frequency (Fig. 9), it was noticed that the amplitudes were largely variable and that the distribution is bimodal, which explains the higher expected peak value.

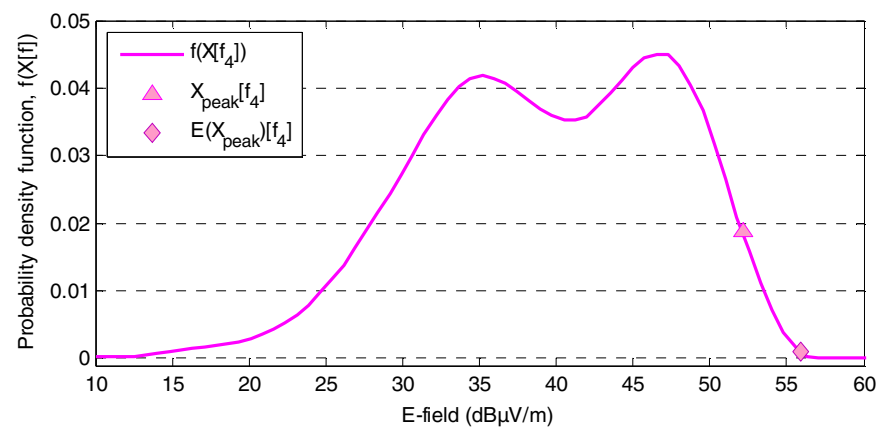

Fig. 9. Estimated probability density functions for the EMI amplitude at $\mathrm{f}_{4}=43.17 \mathrm{MHz}$. 
On the contrary, the estimates obtained by means of the classical Gumbel distribution approximation based on a normality assumption lead to an overestimation of the extreme values, with a mean difference of $7.79 \mathrm{~dB}$ between the expected peak and the measured peak.

\section{CONCLUSION}

A robust statistical estimation of the expected peak EMI value of signal sources which are time varying was achieved following a numerical approach that combines kernel density estimation and Monte Carlo resampling for calculating the parameters of a GEV distribution that model the measured peak emissions as random variables. A key advantage of this approach is that it does not require increasing the dwell time or holding the maximum value over successive frequency sweeps. The experiment confirms this methodology is best suited for calculating the expected maximum peak value of time varying interferences than previous analytical approximations because it does not rely on normality assumptions difficult to ensure in practice.

The proposed technique is an example of how the information provided by full time-domain EMI measurements can be used for obtaining complementary measurement insights helpful in the assessment of the EUT's worst-case emissions that is required for risk and reliability analysis.

In a broader sense, this method could be extended to analyze several factors that influence the EUT's maximum emissions, e.g., the cable layout, turntable rotation, height of the antenna, antenna polarization or even the EUT software configuration Further research is foreseen to allow estimating the distribution function and the maximum EUT's emissions based on larger and more complex data sets comprising the abovementioned sources of variability.

\section{ACKNOWLEDGMENT}

This work was supported in part by the EURAMET 15RPT01 research project (the EMPIR is jointly funded by the EMPIR participating countries within EURAMET and the European Union), by the Spanish "Ministerio de Economía, Industria y Competitividad," under projects TEC2013- 48414C3-3-R and TEC2016-79214-C3-2-R (AEI/FEDER, UE), by the "Secretaria d'Universitats i Recerca del Departament d'Economia i Coneixement de la Generalitat de Catalunya" and the European Social Fund.

\section{REFERENCES}

[1] M. Pous, M. Azpúrua, and F. Silva, "Benefits of full time-domain EMI measurements for large fixed installation," 2016 International Symposium on Electromagnetic Compatibility - EMC EUROPE. pp. 514-519, 2016.

[2] M. A. Azpúrua, M. Pous, S. Çakir, M. Çetintaş, and F. Silva, "Improving Time-Domain EMI measurements through Digital Signal Processing," Electromagn. Compat. Mag. IEEE, vol. 4, no. 2, pp. 66-74, 2015.

[3] M. A. Azpúrua, M. Pous, and F. Silva, "A Measurement System for Radiated Transient Electromagnetic Interference Based on General Purpose Instruments," in Electromagnetic Compatibility (EMC EUROPE), International Symposium on, 2015.

[4] M. A. Azpurua, M. Pous, and F. Silva, "On the Statistical Properties of the Peak Detection for Time-Domain EMI Measurements," IEEE Trans. Electromagn. Compat., vol. 57, no. 6. IEEE, 2015, pp. 1374-1381.

[5] M. Pous, M. A. Azpúrua, and F. Silva, "Measurement and Evaluation Techniques to Estimate the Degradation Produced by the Radiated Transients Interference to the GSM System," IEEE Trans. Electromagn. Compat., vol. 57, no. 6. 2015, pp. 1382-1390.

[6] M. A. Azpurua, M. Pous, F. Silva, and J. A. Oliva, "Fast and Automated Verification of Multi-channel Full Time-Domain EMI measurement systems," in 2017 IEEE International Instrumentation and Measurement Technology Conference (I2MTC), 2017.

[7] IEC CISPR, 16-1-1 ed4.0: Specification for radio disturbance and immunity measuring apparatus and methods - Part 1-1: Radio disturbance and immunity measuring apparatus - Measuring apparatus. IEC, 2015.

[8] C. Keller and K. Feser, "Fast Emission Measurement in Time Domain," IEEE Trans. Electromagn. Compat., vol. 49, no. 4, 2007.

[9] S. L. Marple, Digital Spectral Analysis With Applications. Dover Publications, 2014.

[10] A. Frech, A. Zakaria, S. Braun, and P. Russer, "Ambient noise cancelation with a time-domain EMI measurement system using adaptive filtering," 2008 Asia-Pacific Symposium on Electromagnetic Compatibility and 19th International Zurich Symposium on Electromagnetic Compatibility. pp. 534-537, 2008.

[11] S. Braun, A. Frech, and P. Russer, "CISPR specification and measurement uncertainty of the time-domain EMI measurement system," 2008 IEEE International Symposium on Electromagnetic Compatibility. pp. 1-4, 2008.

[12] S. Braun, T. Donauer, and P. Russer, "A real-time time-domain EMI measurement system for full-compliance measurements according to CISPR 16-1-1," IEEE Trans. Electromagn. Compat., vol. 50, no. 2, pp. 259-267, 2008.

[13] F. Krug and P. Russer, "Quasi-peak detector model for a time-domain measurement system," IEEE Trans. Electromagn. Compat., vol. 47, no. 2, pp. 320-326, 2005.

[14] S. Coles, An Introduction to Statistical Modeling of Extreme Values. Springer London, 2013.

[15] B. Silverman, "Density estimation for statistics and data analysis," Chapman Hall, vol. 37, no. 1, pp. 1-22, 1986.

[16] U. S. Methods, "NIST/SEMATECH, Handbook of Statistical Commerce Department," 2012. 